\title{
Cardiac lipoblastoma of the pulmonary valve: A rare tumour in an unusual location
}

\author{
Feng Tong ${ }^{1}$, Zhangke Guo ${ }^{1}$, and Song Bai $^{2}$ \\ ${ }^{1}$ Beijing Children's Hospital affiliated to Capital Medical University; National Center for \\ Children's HealthChina \\ ${ }^{2}$ Beijing Children's Hospital
}

February 28, 2021

\begin{abstract}
Lipoblastoma is a rare, benign, fatty tissue tumour that occurs in infancy and early childhood. Intrathoracic and mediastinal involvement of this tumour is rare, and an intracardiac location is even rarer. In this study, we report the case of a 15-monthold girl who presented with a tumour in the pulmonary valve. A complete resection of the tumour was undertaken, with a histopathology report confirming the diagnosis of lipoblastoma.
\end{abstract}

\section{Cardiac lipoblastoma of the pulmonary valve: A rare tumour in an unusual location}

Tong Feng, Guo Zhangke, Bai Song

Department of Cardiac surgery, Beijing Children's Hospital affiliated to Capital Medical University

National Center for Children's Health,China

Beijing 100045, China

56 South Lishi Road, Xicheng District, Beijing

Corresponding author: Guo Zhangke, Email: gzkbjetyy@163.comTelephone: +8601069616783

Abstract:Lipoblastoma is a rare, benign, fatty tissue tumour that occurs in infancy and early childhood. Intrathoracic and mediastinal involvement of this tumour is rare, and an intracardiac location is even rarer. In this study, we report the case of a 15-month-old girl who presented with a tumour in the pulmonary valve. A complete resection of the tumour was undertaken, with a histopathology report confirming the diagnosis of lipoblastoma.

Key words: lipoblastoma, pulmonary valve, child

\section{Introduction:}

Lipoblastoma is a rare benign neoplasm of the foetal embryonal white fatty tissue occurring most commonly in infants and young children. It frequently occurs in the subcutaneous tissues of the extremities and the $\operatorname{trunk}^{[1]}$. It can also arise in the abdomen, chest, mediastinum, retroperitoneum, and so on. Primary cardiac lipoblastomas are very rare tumours, and involvement of the pulmonary valve is extremely rare. To our knowledge, this is the first report of such a localization of this type of tumour ${ }^{[2,3]}$.

\section{Case report:}


A 15-month-old girl was admitted to the hospital with a chief complaint of pulmonary artery stenosis found during the foetal period and cardiac murmur found after birth. Her vital parameters were normal; there was no cyanosis, and oxygen saturation $\left(\mathrm{SpO}_{2}\right)$ was $98 \%$ in room air. There was no history of trauma or infection.

Initial examination revealed a systolic murmur at the left sternal edge and clear lung fields, with no lower limb oedema. Transthoracic echocardiography showed a $9.6 \mathrm{~mm}$ x $9.2 \mathrm{~mm}$ nearly circular abnormal cystic mass in the main pulmonary artery adherent to the pulmonary valve (Figure 1(a)). The pulmonary valve appeared thickened and restricted, with a peak gradient of $61 \mathrm{mmHg}$, consistent with valvular pulmonary stenosis. Transthoracic echocardiogram examination also revealed a secundum-type atrial septal defect (ASD) with a diameter measuring $21 \mathrm{~mm}$. In addition, the patient had right heart enlargement and mild tricuspid regurgitation.

The mass was excised through a right thoracotomy under cardiopulmonary bypass. The pulmonary valve orifice was not narrowed, the left anterior valve was lengthy, and the right anterior valve was slightly stiff. The tumour was a sessile cyst with a diameter of $1 \mathrm{~cm}$. It was attached to the left posterior valve and prolapsed into the pulmonary artery. The impression of the surgeon was that the tumour originated from the pulmonary valve, and he could find a clear cleavage (Figure 1(b)). The tumour was completely resected macroscopically. The atrial septal defect was closed by a Gore-Tex patch.

Histological examination of the lesion confirmed the diagnosis of lipoblastoma. Lobules of mature as well as immature fat cells, signet-ring cells, and multivacuolated cells of varying degrees of differentiation can be seen under the microscope. In contrast to myxoid liposarcoma which concentrated peripherally, mature fat cells are concentrated in the central portion of the lobule (Figure 2(a)). Immunohistochemical results showed positive immunostaining for CD34 and S-100 (Figure 2(b)).

The postoperative period was uneventful, and the patient was asymptomatic thereafter. When seen 2 months later, echocardiogram showed no pulmonary valvular incompetence. On further follow-up, there was no recurrence.

\section{Discussion:}

Primary cardiac tumours in childhood have a very low incidence, although in recent years, the incidence has increased because of improvements in heart imaging techniques ${ }^{[3]}[4]$. Most primary cardiac tumours are benign in the sense they are not invasive. However, benign tumours maintain the potential for serious illness related to significant haemodynamic compromise or life-threatening dysrhythmias. In most cases, the first clinical manifestation depends on the tumour location and size. In our case, the mass was not very large in size, and the patient was symptomatic with the persistence of a heart murmur.

Due to the association of lipoblastoma with other heart malformations, preoperative diagnosis remains challenging. Transthoracic echocardiography has high sensitivity for the detection of cardiac masses. It can often provide precise information about the tumour size, location, shape, mobility, and associated features. It is usually the first-line investigation. Cardiac MRI is especially advantageous in defining tissue characteristics and, through the use of gadolinium-based contrast agents, in differentiating between cardiac masses such as tumours, which display variable enhancement, and thrombi, which do not enhance at all ${ }^{[5]}$. Before the operation, this patient was considered to be diagnosed with congenital valvular stenosis and ASD, therefore, no MRI was applied. The diagnosis of lipoblastoma is often made postoperatively. It demonstrated adipocytes in different stages of maturation without anaplasia. The main histological features of the tumour were multinodular and myxoid foci. Chromosomal abnormalities have been reported in lipomatous tumours. In our case, we did not perform any genetic study because the histopathological evaluation alone was definitive.

Tumours on the heart valve are more likely to cause haemodynamic disturbances. Complete resection yields an excellent prognosis ${ }^{[4,6]}$. Occasionally, the resection of adjacent structures is necessary to achieve complete extirpation; however, in our patient, the resection of the adherent pulmonary valve was not required. The resection of the tumour and its adjacent tissue may damage the heart structure; therefore, it is important 
to carefully evaluate preoperative parameters.

Conflicts of interest: On behalf of all authors, the corresponding author states that there is no conflict of interest.

Funding: The study did not receive funding.

Ethical Standards:The study was approved by Beijing children's Hospital Clinical New Technology and Research Ethics Committee.

\section{References:}

[1] Speer A L , Schofield D E, Wang K S , et al. Contemporary management of lipoblastoma[J]. journal of pediatric surgery, 2008, 43(7):0-1300.

[2] Dishop M K, O'Connor W N, Abraham S, et al. Primary Cardiac Lipoblastoma[J]. Pediatric \& Developmental Pathology the Official Journal of the Society for Pediatric Pathology \& the Paediatric Pathology Society, 2001, 4(3):276.

[3] Poonam S , Urmila S , Gita S , et al. Lipoblastoma: an unusual tumour of the left ventricle[J]. European Journal of Cardio-Thoracic Surgery(5):5.

[4] Tzani A , Doulamis I P , Mylonas K S , et al. Cardiac Tumors in Pediatric Patients: A Systematic Review[J]. World Journal for Pediatric \& Congenital Heart Surgery, 2017, 8(5):624-632.

[5] Chen C W , Chang W C, Lee H S, et al. MRI features of lipoblastoma: differentiating from other palpable lipomatous tumor in pediatric patients[J]. Clinical Imaging, 2010, 34(6):453-457.

[6] Mcvay M R , Keller J E , Wagner C W, et al. Surgical management of lipoblastoma[J]. journal of pediatric surgery, 2006, 41(6):0-1071.
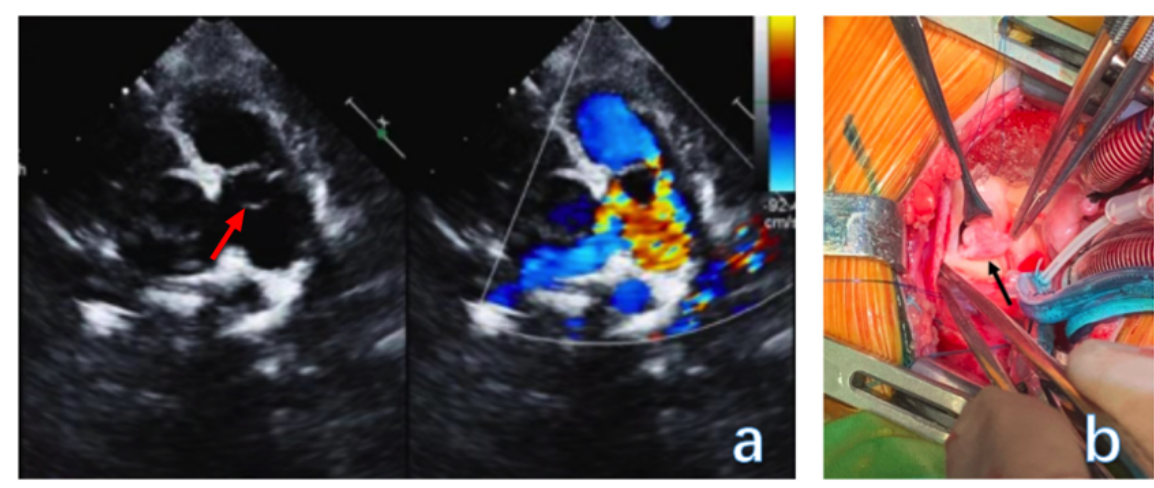

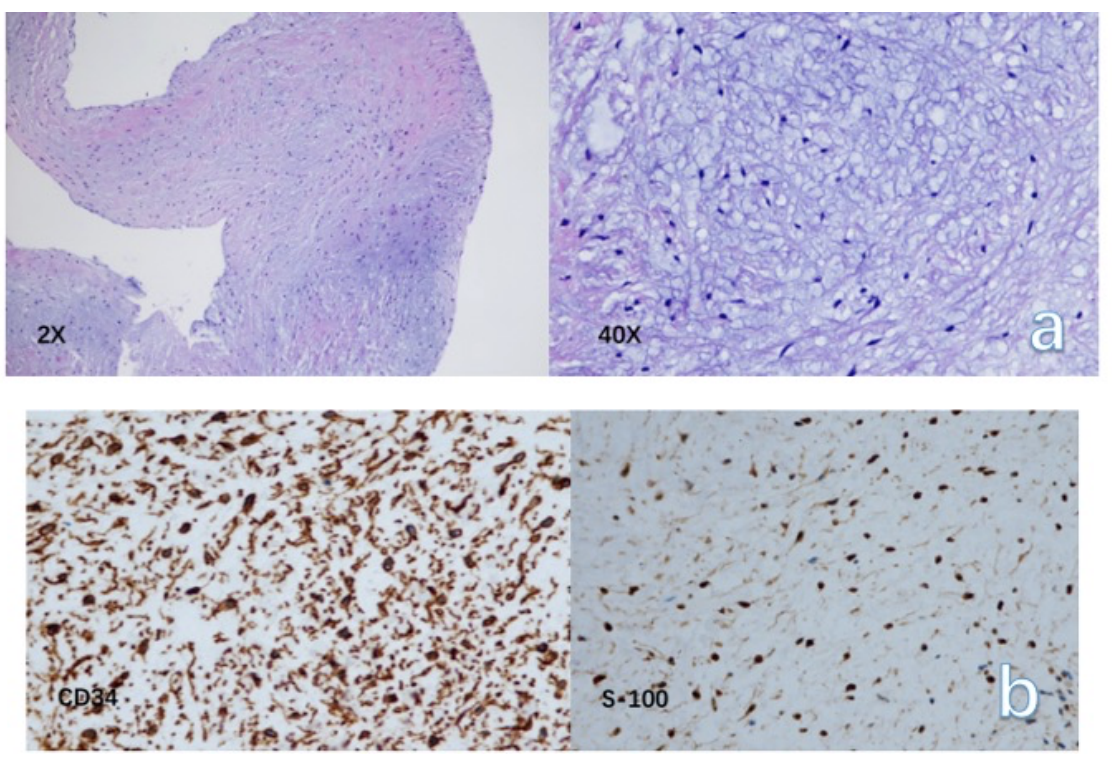